\title{
Anatomic Double-Bundle Medial Patellofemoral Ligament Reconstruction with Autologous Semitendinosus: Aperture Fixation Both at the Femur and the Patella
}

\author{
Michele Vasso ${ }^{1}$ Katia Corona ${ }^{2}$ Giuseppe Toro ${ }^{1}$ Marco Rossini ${ }^{1}$ Alfredo Schiavone Panni ${ }^{1}$
}

${ }^{1}$ Multidisciplinary Department of Medico-Surgical and Dentistry Specialties, “Luigi Vanvitelli” University, Naples, Italy

2 Department of Medicine and Health Sciences, University of Molise, Campobasso, Italy

Joints 2017;5:256-260.

\begin{abstract}
Address for correspondence Katia Corona, MS, Department of Medicine and Health Sciences, University of Molise, Via Francesco De Sanctis, 86100, Campobasso, Italy (e-mail: katiacorona@tiscali.it).
\end{abstract}

\begin{abstract}
Keywords

- medial patellofemoral ligament

- reconstruction

- double-bundle

- aperture fixation

- semitendinosus

Medial patellofemoral ligament (MPFL) represents the main restraint against lateral patellar displacement. The MPFL insertion at the patella is up to $30 \mathrm{~mm}$ wide and is located along the upper half of the medial patellar rim. The femoral insertion of the MPFL is approximately $10 \mathrm{~mm}$ distal to the apex of the adductor tubercle and $16 \mathrm{~mm}$ proximal to the medial epicondyle. Since most of the patients suffer from MPFL rupture just after the first patellar dislocation, MPFL reconstruction results necessarily in all cases of chronic objective patellar instability to reestablish the primary passive patellofemoral stabilizer. Over time, different techniques of MPFL reconstruction have been proposed with promising results in terms of patient satisfaction and redislocation rate. However, each of these techniques may present peculiar problems and/or complications. An anatomic double-bundle MPFL reconstruction through an aperture fixation both at the femur and at the patella is here presented. The anatomic double-bundle MPFL reconstruction could allow recreating the fan-shape and biomechanics of original MPFL, whereas aperture fixation could provide a strong and safe fixation without risk of loosening or slackening of the graft.
\end{abstract}

\section{Introduction}

The medial patellofemoral complex consisting of the medial patellofemoral ligament (MPFL) and the medial patellotibial ligament represents the main passive stabilizer of the patellofemoral (PF) joint. In particular, the MPFL represents the main restraint against lateral patellar displacement. ${ }^{1}$ The MPFL insertion at the patella is up to $30 \mathrm{~mm}$ wide and is located along the upper half of the patella. Proximal fibers are mainly attached to the vastus intermedius, without tight adhesion to the vastus medialis. Distal fibers are interdigitated with the deep layer of the medial retinaculum. ${ }^{2}$ The femoral insertion of the MPFL is approximately $10 \mathrm{~mm}$ distal to the apex of the adductor tubercle and $16 \mathrm{~mm}$ proximal to the medial epicondyle. $^{3}$ MPFL is located in layer 2 of the medial soft-tissue structures between medial retinaculum (layer 1 ) and knee capsule (layer 3). Clinically, up to 94 to $100 \%$ of patients suffer from MPFL rupture just after the first patellar dislocation, and approximately half of these patients will develop recurrent dislocations. ${ }^{4}$ For this reason, MPFL reconstruction is necessary in all cases of chronic objective patellar instability, to reestablish the primary passive PF stabilizer. However, MPFL reconstruction should be supplemented with additional procedures to address various aspects of PF dysplasia when present: severe trochlear dysplasia, patella alta, patellar tilt, and lateralization of the tibial tuberosity.

Numerous techniques for MPFL reconstruction have been described, and they all aim to supply tendon graft from the medial aspect of the patella to the femoral insertion site of the native MPFL. Numerous graft sources for reconstruction published online October 4, 2017
DOI https://doi.org/ $10.1055 / \mathrm{s}-0037-1607192$ ISSN $2282-4324$
Copyright (c) 2017 Georg Thieme Verlag License terms KG Stuttgart · New York 
are described in the literature, including semitendinosus, gracilis, partial quadriceps, partial patellar tendon, partial adductor tendon, vastus medialis retinaculum, allografts or artificial grafts. Similarly, different techniques and devices for fixing the graft have been used, especially at the patellar MPFL attachment site: bone tunnels, transosseous sutures, small interference screws and suture anchors. However, each of these techniques could present peculiar problems and complications, and biomechanical alterations on the PF joint. Anatomical restoration of MPFL should be the main goal of every reconstruction technique. In this regard, double-bundle MPFL reconstruction at the patellar side could restore native MPFL morphology and biomechanics, therefore, providing more patellar stability, both in extension and up to the first 60 to 70 degrees of flexion. ${ }^{2}$ Likewise, direct aperture fixation could provide firm fixation, thus reducing the risk of loosening or slackening of the graft. ${ }^{5}$

This technical note aimed to present an anatomical double-bundle MPFL reconstruction through an aperture fixation both at the femur and at the patella. The autologous semitendinosus was always chosen for this procedure. The anatomical double-bundle reconstruction with aperture fixation described here represents a modification of the technique initially introduced by Schöttle et al. ${ }^{6}$

\section{Surgical Technique}

Before the surgery, an examination under anesthesia is performed to confirm the diagnosis of patellar instability and hypermobility. Arthroscopy is routinely performed to treat, if necessary, any intra-articular lesions and evaluate patellar tracking throughout knee motion.

\section{Semitendinosus Graft Preparation}

A 2-cm vertical skin incision is made over the insertion site of the pes anserinus. After incising the sartorius aponeurosis, the semitendinosus tendon is harvested. The two ends of the tendon graft are each sutured with no. 2 absorbable sutures using a whipstitch technique up to approximately $2 \mathrm{~cm}$ from each end. We routinely use the semitendinosus graft, since in patients with a short gracilis this tendon may not be long enough to reach the anatomical femoral insertion.

\section{MPFL Insertion at the Patella}

A 2-cm medial parapatellar skin incision is performed along the proximal half of the patella, where the patellar MPFL insertion is located. Retinaculum and periosteum of the medial patella rim are incised longitudinally and elevated off the bone. Two guidewires are drilled into the patella at the proximal and distal ends of the upper half of the medial edge; the two guidewires slightly converge laterally The guidewires are subsequently overdrilled with a cannulated 4-mm drill to a depth of $20 \mathrm{~mm}$. The tails of one graft end are passed through the eyelet of the first $4.75-\mathrm{mm}$ fully threaded, knotless anchor (SwiveLock; Arthrex, Naples, Florida, United States) and the graft/anchor is pushed into the proximal drill hole until the eyelet is fully seated. While maintaining tension on the suture limbs, the SwiveLock anchor is screwed into the patella. After removal of the driver, the SwiveLock suture should be removed or tied to the graft sutures to reinforce the fixation. This procedure is then repeated for the second graft end. The two free sutured graft ends are thus fixed into the patellar holes achieving an anatomical graft fixation. A double-bundle direct aperture fixation at the patellar side is thus achieved leaving the graft loop free (-Fig. 1). After successive fixation of the graft at the femoral MPFL insertion, the reflected periosteum and medial retinaculum were sutured on top of the graft for additional fixation.

\section{MPFL Insertion at the Femur}

For an anatomical femoral insertion of the graft, the medial epicondyle and adductor tubercle are palpated. A $1-\mathrm{cm}$ longitudinal incision is made in this area, and a $2-\mathrm{mm}$ guidewire with an eyelet is placed at the correct femoral MPFL insertion point. The guidewire placement is controlled by an image intensifier on a straight lateral view to obtain and identify the anatomical femoral insertion point. It has been shown ${ }^{7}$ to be located $1 \mathrm{~mm}$ anterior to an elongation of the posterior femoral cortex in between the posterior articular border of the medial femoral condyle and the most proximal point of the Blumensaat line (-Fig. 2). At this point, the isometry of the MPFL may be evaluated by maintaining adequate tension on the graft and cycling the knee through the full range of motion (ROM). When the isometry
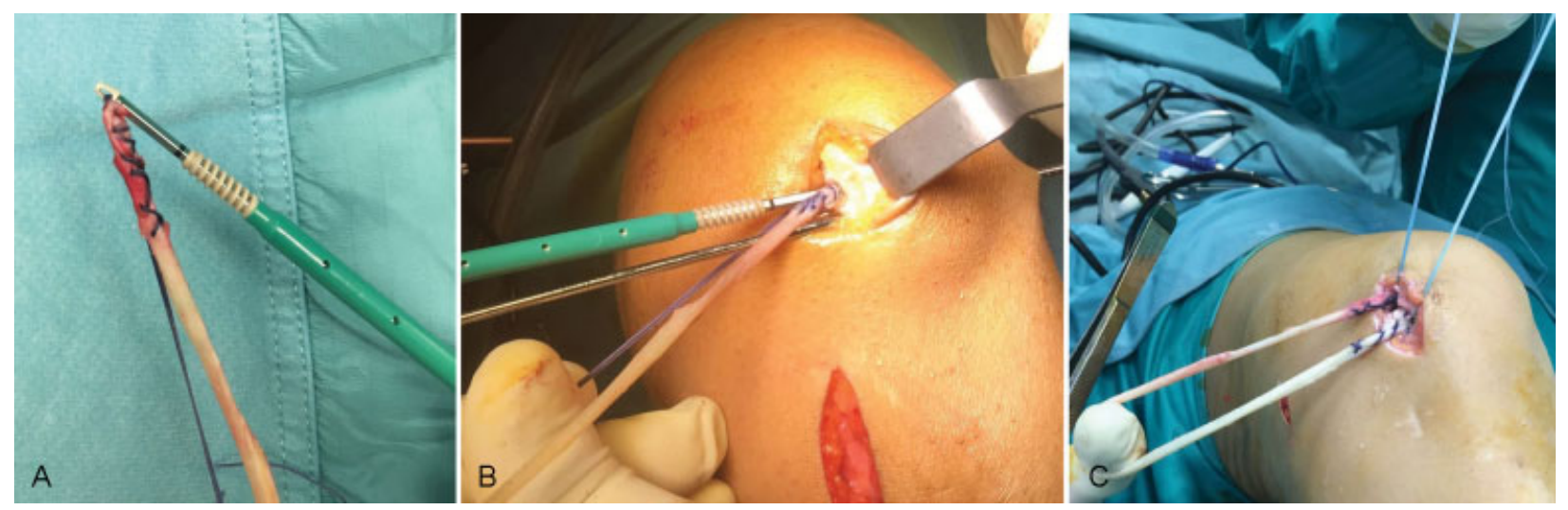

Fig. 1 The graft sutures are pulled through the eyelet of the SwiveLock (Arthrex, Naples, Florida, United States) (A), and pushed into the drill holes (B). Keeping the suture under tension, the graft ends are fixed with the SwiveLock anchor leaving the graft loop free (C). 


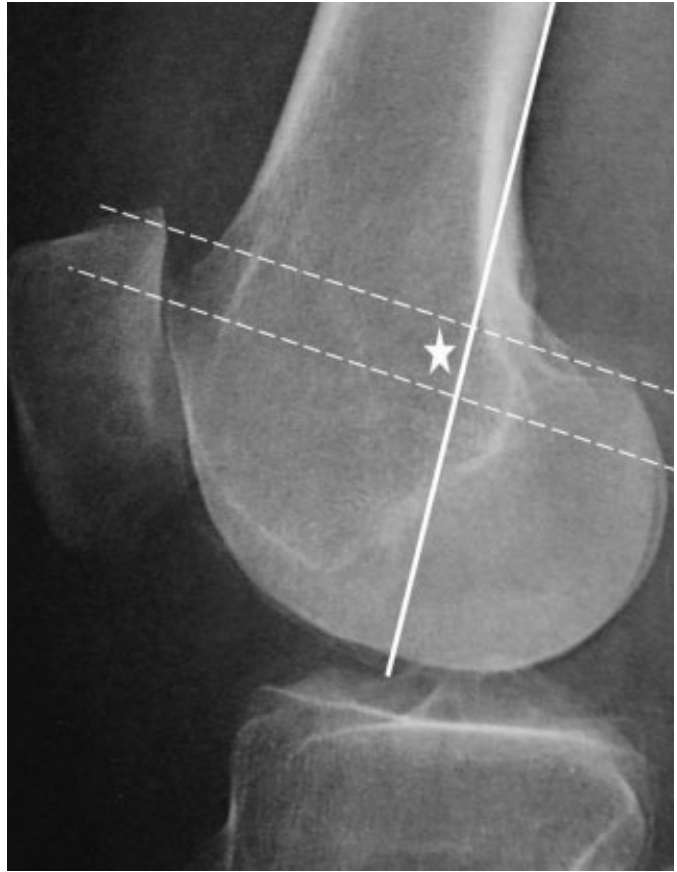

Fig. 2 Straight lateral view of the knee is obtained intraoperatively with an image intensifier. The radiographic landmark of the femoral attachment site is determined by three lines: an elongation of the posterior femoral cortex (continuous line) and two lines perpendicular to this line (dashed lines), one passing through the posterior articular border of the medial femoral condyle and one passing through the most proximal aspect of the Blumensaat line. The landmark is just anterior ( $1 \mathrm{~mm}$ ) to the continuous line, in the midpoint between the dashed lines (asterisk).

is respected, then the guidewire is drilled (directed slightly anterior to avoid posterior soft-tissue injuries) and pushed outside the lateral side of the knee. At this point, a halftunnel with a diameter $1 \mathrm{~mm}$ larger than that of the double semitendinosus is created with a cannulated drill. The length of the femoral half-tunnel depends on the length of the double semitendinosus. The guidewire is maintained in the femur, as it will be used to pass the graft into the femur.

At this point, a blunt dissection is performed to create a soft-tissue tunnel in layer 2 of the medial soft-tissue structures from the patellar insertion point to the femoral insertion point, deep to the superficial medial retinaculum (layer 1) but superficial to the synovium (layer 3), being careful to leave the capsule intact. A no. 2 suture loop is passed from the femoral side to the patellar one through this space using an angled clamp (-Fig. 3). The suture loop is used to carry the graft from the patellar side to the femoral insertion point (-Fig. 4). The suture shuttle used to pull the graft is then inserted into the eyelet of the femoral guidewire (-Fig. 5), and the graft is successively passed through the femoral tunnel by pulling the guidewire and the suture shuttle out of the lateral side of the knee. Equal tension on both graft bundles is maintained during graft passage into the femoral half-tunnel. Femoral fixation is achieved with a biodegradable interference screw $1 \mathrm{~mm}$ larger than the graft, which is passed over a nitinol guidewire. Femoral fixation is per-

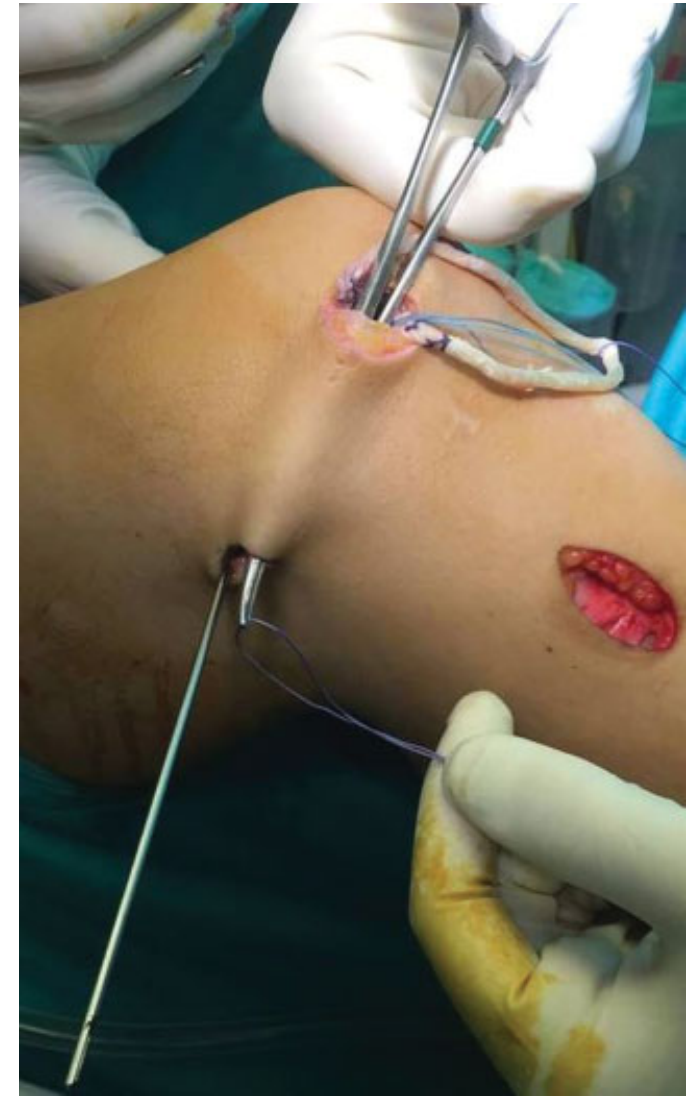

Fig. 3 As the MPFL is situated in the second layer of the medial patellofemoral complex, a tunnel between the superficial medial retinaculum and the joint capsule is created, and an angled clamp is brought into this space and directed toward the femoral MPFL insertion, cautiously avoiding any injury to the joint. A suture loop is then inserted into this tunnel using the angled clamp. MPFL, medial patellofemoral ligament.

formed in 30 degrees of knee flexion ${ }^{8}$ with the lateral patellar edge positioned in line with the lateral trochlear border. A traditional skin closure is thus performed.

\section{Postoperative Care}

Rehabilitation involves a knee brace for protection. Flexion is restricted to 60 degrees for the first two postoperative weeks and to 90 degrees for the third postoperative week; successively, a full ROM is allowed. Partial weight-bearing with crutches is allowed during the first 3 weeks, then full weight-bearing without crutches is regained. Leg raising and quadriceps isometric strength exercises are immediately performed. Noncontact sports are allowed after 3 months, while contact sports can be resumed 6 months after surgery.

\section{Discussion}

This technical note aimed to describe the procedure for an anatomical double-bundle reconstruction of the MPFL, respecting not only the original ligament shape but also the anatomical patellar and femoral insertion areas. Moreover, 


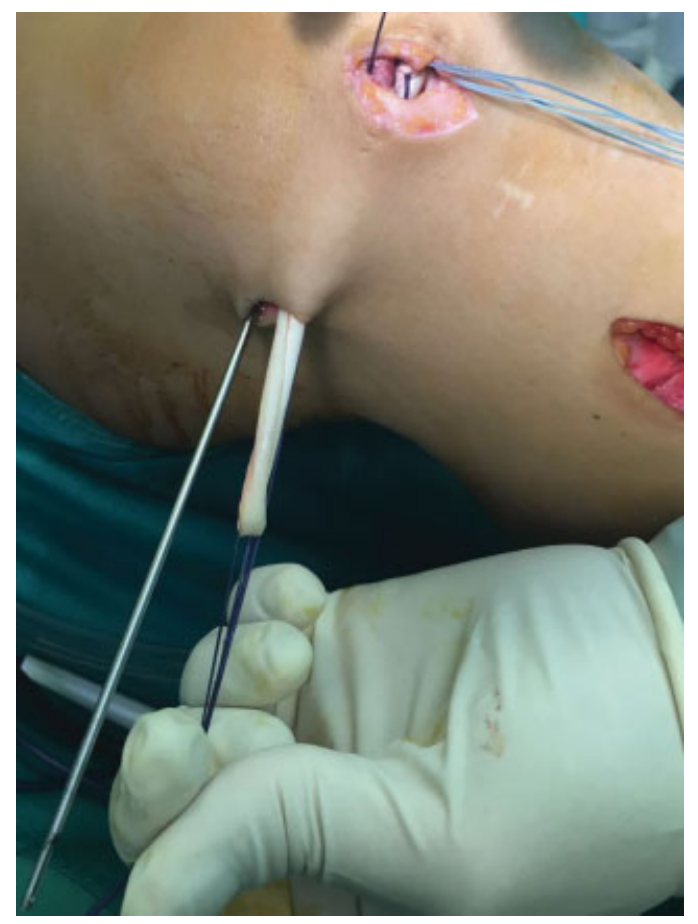

Fig. 4 The suture loop is carried by the curved clamp at the medial patellar border and used to pull the graft from the patellar side to the femoral insertion point.

an aperture fixation was assured both at the femur and at the patella. The procedure presented here represents a modification of the technique initially introduced by Schöttle et al. ${ }^{6}$ The main difference of this procedure compared with the Schöttle's one was that semitendinosus was used instead of gracilis, since in patients with a short gracilis this tendon

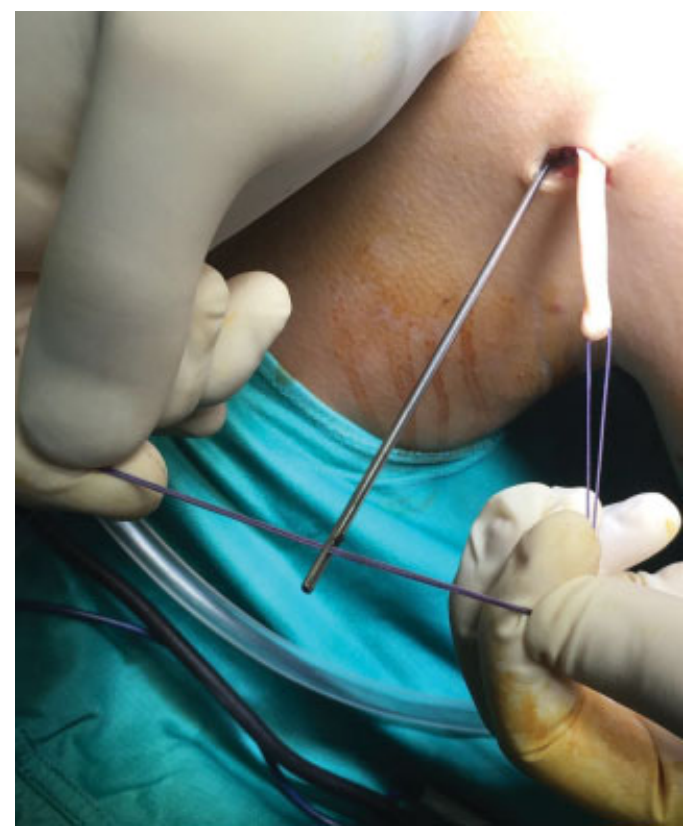

Fig. 5 The suture shuttle used to pull the graft from the patellar to the femoral side is inserted into the eyelet of the femoral guidewire and used to pull the graft through the femoral tunnel. may not be long enough to reach the anatomical femoral insertion, especially when an anatomic double-bundle reconstruction with aperture fixation devices are performed. Also, after graft harvesting, the proximal half of the patella is immediately prepared to fix the two free ends of the graft with the SwiveLock anchors; only at this point, the detection and the dissection of the layer 2 of the medial soft tissues is performed, being that facilitated by a better visualization of the structures of the medial retinaculum due to the previous medial patellar side preparation. Finally, by fixing the graft to the patella before preparing the femoral insertion site, the isometry of the MPFL may be assessed to detect the most anatomical femoral insertion.

During years, multiple grafts and fixation techniques have been proposed to reconstruct the MPFL with promising clinical results and low redislocation rate, but each of these techniques may present potential problems and/or complications. The tendon transfer procedure with patellar or quadriceps grafts presents the clear disadvantage of being a single-bundle reconstruction, with the evident risk of increasing the rotational moment of the patella during knee motion. Moreover, when using the patellar tendon transfer procedure, the patella insertion cannot be reconstructed at its anatomical insertion. Also, the transferred muscle may be weakened in its original strength. ${ }^{8}$ Finally, the single-bundle MPFL reconstruction could not restore normal patellar tracking at any flexion angle. ${ }^{9}$ Although no previous studies confirmed that double-bundle MPFL reconstruction is better in morphology and biomechanics than quadriceps tendon transfer, it is widely recognized how the patellar attachment of the MPFL could vary, from the proximal half of the patella to even the upper two-thirds of the patella. ${ }^{2}$ Therefore, sometimes quadriceps tendon cannot restore the anatomy of the native MPFL. Furthermore, Mochizuki et $\mathrm{al}^{2}$ recently demonstrated the presence of two bundles in the patellar attachment of MPFL (proximal and distal fibers), thus concluding that MPFL reconstruction by recreating both bundles might better restore ligament function and improve patellar stability.

We primarily performed a double-bundle MPFL reconstruction with two divergent transverse tunnels on the patella. ${ }^{10}$ However, it has been largely proven that most of the techniques using transverse patellar tunnels increase the risk of patellar fracture. ${ }^{11}$ Anatomical patellar fixation by looping the graft through short bone tunnels at the medial edge of the patella, without using fixation devices, has been proposed to reduce the risk of fracture; however, tunnel widening might occur in the long-term, thus causing graft loosening. In general, nonapertured fixation techniques could cause loosening, slippage or slackening of the graft. Patellar graft fixation into a bony rim with suture anchors or by tying graft sutures at the lateral patellar edge may potentially result in graft slippage and/or loosening. ${ }^{6}$ Similarly, fixing the graft to the surrounding soft tissues might cause graft loosening and/or slackening. Looping the graft through a single small patellar tunnel has been proposed to avoid fractures, with promising results. ${ }^{12}$ However, if micromotion in the patellar tunnels develops it may lead to graft 
slackening; moreover, if the graft is not long enough, the femoral insertion cannot be reached. For all these reasons, we adopted the abovementioned anatomical double-bundle MPFL reconstruction through aperture fixation both at the femur and at the patella. This technique could not only recreate the fan-shape of MPFL but also respect the anatomical patellar and femoral insertion areas. Also, the direct aperture fixation both at the femoral and patellar sides could provide firm fixation and minimize the risk of patella fracture. ${ }^{5}$ Finally, although different knee flexion angles have been described for MPFL graft fixation, varying from 20 to 70 degrees, $^{13}$ several studies showed that MPFL has its maximal length and restraint against patella lateralization at 30 degrees of knee flexion. For this reason, we fixed the graft at 30 degrees of knee flexion and given the strength of aperture fixation we allowed a gradual regaining of knee flexion up to 90 degrees within the first 3 weeks, without reporting any complication.

\section{References}

1 Panni AS, Cerciello S, Vasso M. Patellofemoral instability: surgical treatment of soft tissues. Joints 2013;1(01):34-39

2 Mochizuki T, Nimura A, Tateishi T, Yamaguchi K, Muneta T, Akita K. Anatomic study of the attachment of the medial patellofemoral ligament and its characteristic relationships to the vastus intermedius. Knee Surg Sports Traumatol Arthrosc 2013;21(02): 305-310

3 Wijdicks CA, Griffith CJ, LaPrade RF, et al. Radiographic identification of the primary medial knee structures. J Bone Joint Surg Am 2009;91(03):521-529
4 Panni AS, Vasso M, Cerciello S. Acute patellar dislocation. What to do? Knee Surg Sports Traumatol Arthrosc 2013;21(02):275-278

5 Hapa O, Akşahin E, Özden R, et al. Aperture fixation instead of transverse tunnels at the patella for medial patellofemoral ligament reconstruction. Knee Surg Sports Traumatol Arthrosc 2012; 20(02):322-326

6 Schöttle PB, Hensler D, Imhoff AB. Anatomical double-bundle MPFL reconstruction with an aperture fixation. Knee Surg Sports Traumatol Arthrosc 2010;18(02):147-151

7 Schöttle PB, Romero J, Schmeling A, Weiler A. Technical note: anatomical reconstruction of the medial patellofemoral ligament using a free gracilis autograft. Arch Orthop Trauma Surg 2008; 128(05):479-484

8 Amis AA, Firer P, Mountney J, Senavongse W, Thomas NP. Anatomy and biomechanics of the medial patellofemoral ligament. Knee 2003;10(03):215-220

9 Parker DA, Alexander JW, Conditt MA, Uzodinma ON, Bryan WJ. Comparison of isometric and anatomic reconstruction of the medial patellofemoral ligament: a cadaveric study. Orthopedics 2008;31(04):339-343

10 Panni AS, Alam M, Cerciello S, Vasso M, Maffulli N. Medial patellofemoral ligament reconstruction with a divergent patellar transverse 2-tunnel technique. Am J Sports Med 2011;39(12): 2647-2655

11 Schiphouwer L, Rood A, Tigchelaar S, Koëter S. Complications of medial patellofemoral ligament reconstruction using two transverse patellar tunnels. Knee Surg Sports Traumatol Arthrosc 2017;25(01):245-250

12 Zanon G, Marullo M, Benazzo F. Double-bundle medial patellofemoral ligament reconstruction with a single patellar tunnel. Arthrosc Tech 2013;2(04):e401-e404

13 Kang HJ, Cao JH, Pan S, Wang XJ, Yu DH, Zheng ZM. The horizontal Y-shaped graft with respective graft tension angles in anatomical two-bundle medial patellofemoral ligament reconstruction. Knee Surg Sports Traumatol Arthrosc 2014;22(10):2445-2451 